\title{
Repetophragma calongeii sp. nov. and other interesting dematiaceous hyphomycetes from the North of Spain
}

\author{
by \\ Carolina Silvera-Simón ${ }^{1}$, Julio Mena-Portales², Josepa Gené1 ${ }^{1}$ Josep Cano ${ }^{1} \&$ Josep Guarro $^{1}$ \\ ${ }^{1}$ Unitat de Microbiologia, Facultat de Medicina i Ciències de la Salut, Universitat Rovira i Virgili, E-43201 Reus, España. \\ carolina.silvera@estudiants.urv.cat, josepa.gene@urv.cat, josep.cano@urv.cat,josep.guarro@urv.cat \\ ${ }^{2}$ Instituto de Ecología y Sistemática, Ministerio de Ciencia, Tecnología y Medio Ambiente, AP 8029, \\ CP 10800, Ciudad de la Habana, Cuba. julio.mena@infomed.sld.cu
}

\begin{abstract}
Silvera-Simón, C., Mena-Portales, J., Gené, J., Cano, J. \& Guarro, J. 2009. Repetophragma calongeii sp. nov. and other interesting dematiaceous hyphomycetes from the North of Spain. Anales Jard. Bot. Madrid 6651: 33-39.

Repetophragma calongeii sp. nov., a dematiaceous hyphomycete found on unidentified dead wood collected in the Pagoeta Natural Park, Spain, is described and illustrated. The fungus is recognized by numerous percurrent proliferations in the conidiophores and its 12-27 euseptate, subcylindrical or subfusiform conidia. A key to species of Repetophragma without rostrate conidia is given and 18 additional dematiaceous hyphomycetes are firtly reported for Spain.
\end{abstract}

Keywords: anamorphic fungi, plant debris, taxonomy.

\section{Introduction}

During a survey of plant debris from the North of Spain some interesting dematiaceous hyphomycetes were found. Among them several species are reported for de first time from the Iberian Peninsula and a new species of Repetophragma is proposed, which was named as a tribute to Prof. Francisco de Diego Calonge, from the Real Jardín Botánico de Madrid, on the anniversary of his 70th birthday and for his excellent contribution to mycology.

\section{Material and methods}

The samples were collected from different Natural Parks located mainly in Euskadi and Asturias (North of Spain), put into sterilized polyethylene bags and

\section{Resumen}

Silvera-Simón, C., Mena-Portales, J., Gené, J., Cano, J. \& Guarro, J. 2009. Repetophragma calongeii sp. nov. y otro interesante hifomicete dematiáceo del norte de España. Anales Jard. Bot. Madrid 6651: 33-39 (en inglés).

Se describe e ilustra un hifomicete dematiáceo, Repetophragma calongeii sp. nov., encontrado sobre madera muerta colectada en el Parque Natural de Pagoeta, España. Este hongo se caracteriza por presentar conidióforos con numerosas proliferaciones percurrentes y conidios subcilíndricos o subfusiformes, 12-17 euseptados. Se propone una clave dicotómica para las especies de Repetophragma que no presentan conidios rostrados o apendiculados. Además, se describen otras 18 especies de hifomicetes dematiáceos que son nuevas citas para el catálogo micológico español.

Palabras clave: hongos anamórficos, restos vegetales, taxonomía.

stored in the refrigerator at $4-7^{\circ} \mathrm{C}$ in the laboratory until they were processed. The plant material was placed into sterile moist chambers, which were incubated at room temperature $\left(22-25^{\circ} \mathrm{C}\right)$ and examined periodically under a stereomicroscope over a 2 -month period. Microscopic features of the fungi growing on the natural substratum were examined from wet mounts prepared in $85 \%$ lactic acid under light microscopy. Drawings were made using a Camara Lucida (1.25×, Olympus). Micrographs were obtained by light microscopy (Leiz Dialux 20) and by scanning electron microscopy (Jeol JSM-6400).

\section{Taxonomic treatment}

Repetophragma calongeii J. Mena, Silvera \& Gené, sp. nov. (Figs. 1, 2). 
Etym.: named as a tribute to the excellent mycologist F. de Diego Calonge.

Coloniae effusae, brunneae vel atro brunneae, pilosae, saepe incospicuae. Mycelium plerumque in substrato immersum. Conidiophora macronematosa, monematosa, solitaria, simplicia, recta vel leviter flexuosa, septata, brunnea vel atro brunnea, laevia, 32-75 ×5-7 $\mu \mathrm{m}$. Cellulae conidiogenae monoblasticae in conidiophoris incorporatae, terminales, brunneae vel atro brunneae, cylindricae, 5-5.8 um latae, cum 4-9 proliferationis percurrentes. Conidia acrogena, solitaria, sicca, recta, brunnea vel modice brunnea, laevia, 17-27 euseptata, subcylindrica vel subfusiformia, 80-165 × (8)9-10.5 $\mu \mathrm{m}, 5-7(8) \mu \mathrm{m}$ lata ad apicem, 5-6 um lata ad basim.

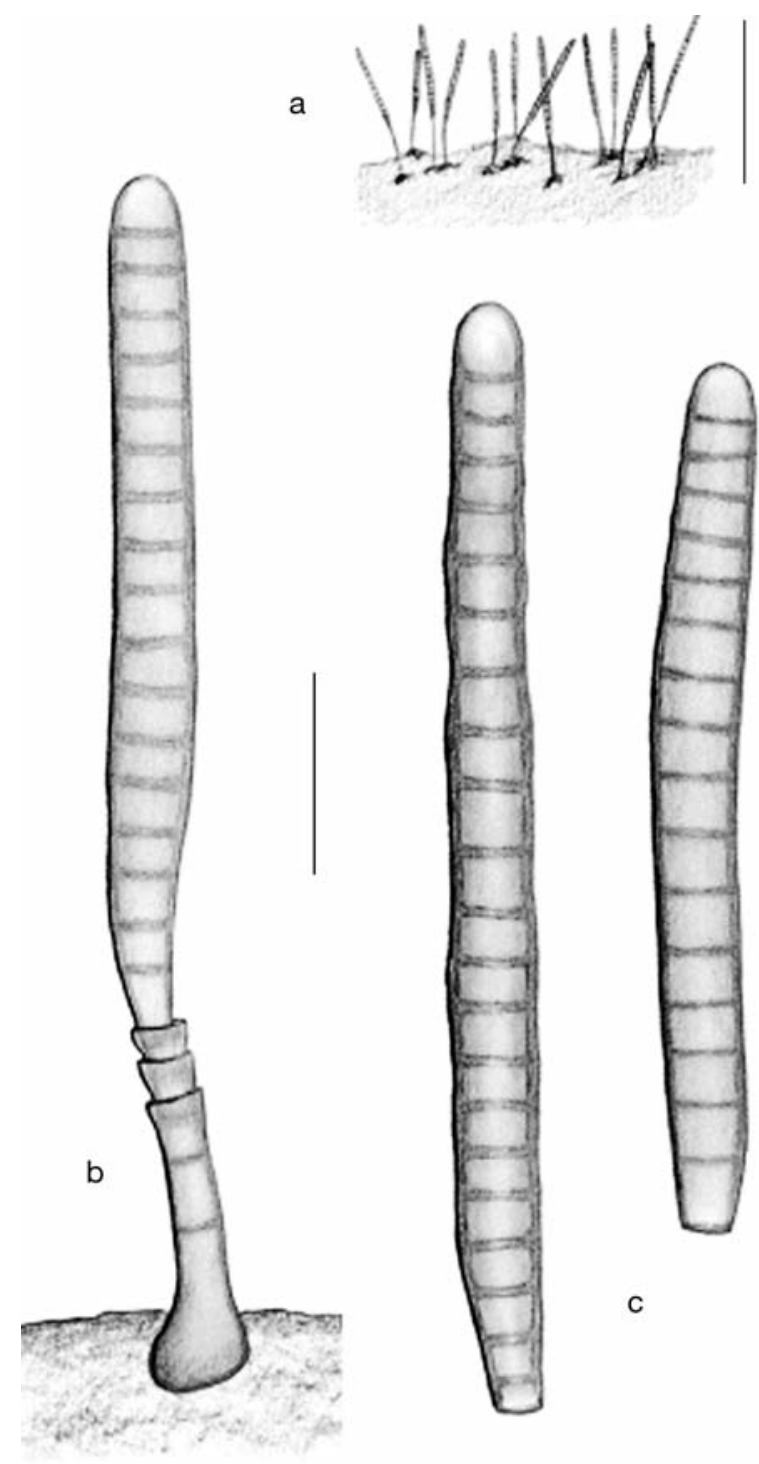

Fig. 1. Repetophragma calongeii, IMI 396700: a, sketch habit; b, c, a conidiophore with annellidic percurrent proliferations and conidia. Scale bars: $a=200 \mu \mathrm{m} ; \mathrm{b}, \mathrm{c}=25 \mu \mathrm{m}$.
Colonies effuse, brown to dark brown, hairy, often inconspicuous. Mycelium mostly immersed in the substratum. Conidiophores macronematous, mononematous, solitary, unbranched, straight or slightly flexuous, septate, brown to dark brown, smooth-walled, cylindrical, 32-75 × 5-7 $\mu \mathrm{m}$. Conidiogenous cells monoblastic, integrated, terminal, mid brown to dark brown, cylindrical, 5-5.8 $\mu \mathrm{m}$ wide, with 4-9 annellidic, percurrent proliferations. Conidia acrogenous, solitary, dry, straight, brown to mid brown, smooth-walled, 12-27 euseptate, subcylindrical or subfusiform, 80-165 $\times$ (8)9-10.5 $\mu \mathrm{m}$ at the broadest part, apex rounded, 5-7(8) $\mu \mathrm{m}$ wide, base truncate, 5-6 $\mu \mathrm{m}$ wide.

SPAIN. Guipúzcoa: Pagoeta Natural Park, IV2008, on dead wood, J. Mena and C. Silvera (holotype IMI 396700; isotype FMR 10089).

The genus Repetophragma Subram., with R. biseptata (M.B. Ellis) Subram. as type species, was segregated from Sporidesmium Link by Subramaniam (1992) to accommodate those species which presented conidiogenous cells with annellidic percurrent proliferations and euseptate conidia. Under these morphological criteria and according to Index Fungorum (http://indexfungorum.org/names/names.asp) a total of 18 species are included in Repetophragma. In a recent molecular phylogenetic study, Shenoy \& al. (2006) demonstrated that some species of Repetophragma as well as other members of Sporidesmium-like genera were clearly polyphyletic; they were dispersed into different families and orders of Sordariomycetes and Dothideomycetes. On the basis of Shenoy \& al. findings (2006), Iturriaga \& al. (2008) recently described a fungus, Sporidesmium lichenicola Iturr., D. Hawksw. \& J.L. Crane, despite its morphological similarity with Repetophragma, and transferred Repetophragma sinense W.P. Wu to Sporidesmium. It is evident that the morphology is not enough to clarify the natural relationships among Sporidesmium-like fungi and new molecular studies involving more species will be necessary to build a robust taxonomy for those fungi. Therefore, until the taxonomy of the these fungi is resolved, considering that most species accepted in Repetophragma have euseptate conidia with rounded well defined apices, without rostrate or appendiculate apical cell and annellidic percurrent proliferartion of the conidiogenous cells (Iturriaga \& al., 2008), we prefer to described the new species in Repetophragma. The only species of Repetophragma morphologically similar to R. calongeii is R. goidanichii (Rambelli) W.P. Wu (Wu \& Zhuang, 2005), but the latter differs by its shorter and less septate conidia (Fig. 18). A dichotomous key to differentiate these species of Repetophragma that lacks conidia with apical beaks or appendages is given below. 


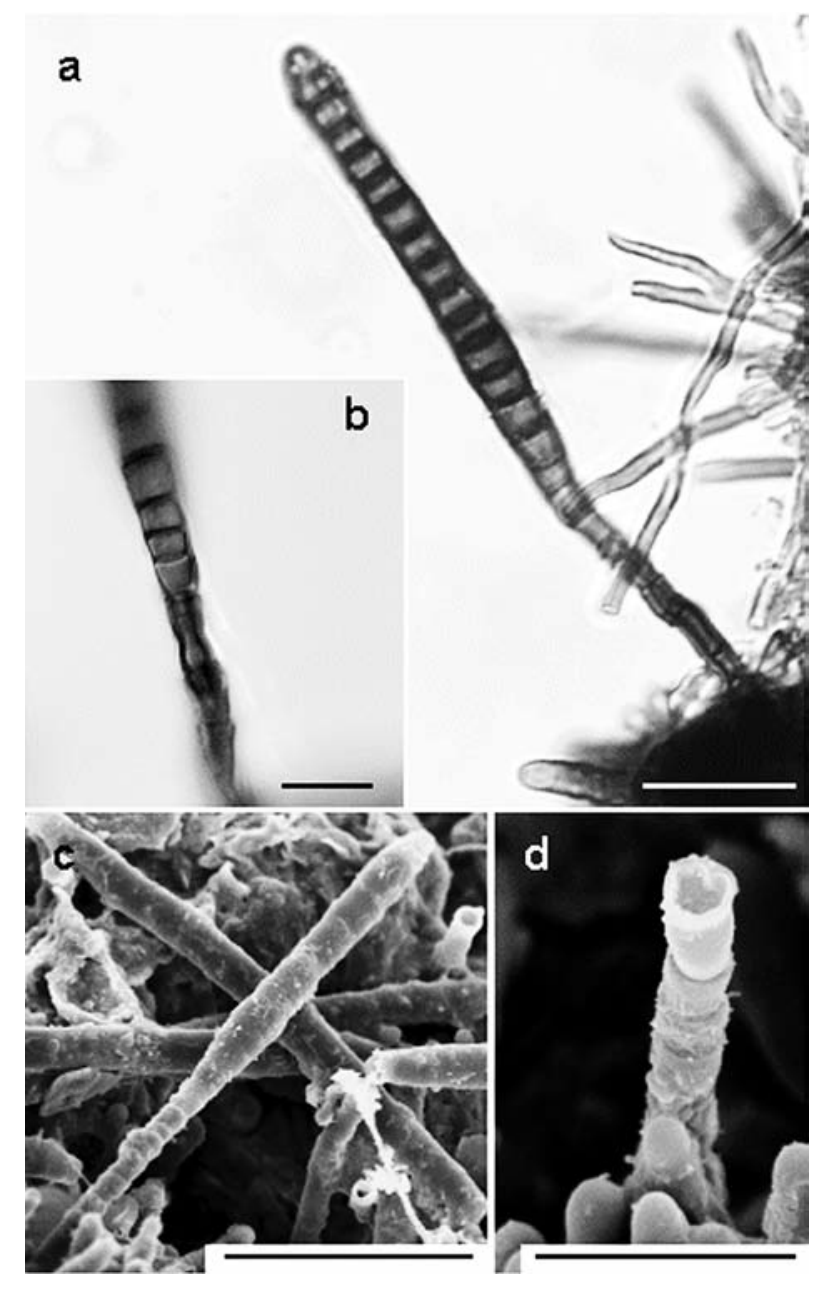

Fig. 2. Repetophragma calongeii, IMI 396700: a, c, conidiophores with annellidic percurrent proliferations producing conidia; $\mathbf{b}, \mathbf{d}$, detail of the percurrent proliferations. Scale bars: a, c = $50 \mu \mathrm{m} ; \mathrm{b}=10 \mu \mathrm{m} ; \mathrm{d}=20 \mu \mathrm{m}$.

KEY TO SPECIES OF REPETOPHRAGMA WITHOUT ROSTRATE OR APPENDICULATE CONIDIA

1. Conidia more than $15 \mu \mathrm{m}$ wide, $100-140 \times 15-20 \mu \mathrm{m}$.

1. Conidia narrower, up to $15 \mu \mathrm{m}$ wide ............ 2

2. Conidia up to 4 septate . . . . . . . . . . . . . . 3

2. Conidia more than 4 septate . . . . . . . . . . . . . . 4

3. Conidia navicular, 2 septate, $17-23 \times 7-8 \mu \mathrm{m}$. .R. biseptatum

3. Conidia mostly ellipsoidal, 2-3 septate, 22-33 $\times 8-12 \mu \mathrm{m}$. .

4. Conidia more than $12 \mu \mathrm{m}$ wide, $40-60 \times 12-15 \mu \mathrm{m}$

R. dennisii

R. cambrense

4. Conidia up to $12 \mu \mathrm{m}$ wide . . . . . . . . . . . . . 5

5. Conidia more than 8 septate . . . . . . . . . . . 6

5. Conidia up to 8 septate . . . . . . . . . . . . . . . 7

6. Conidia 8-10 septate, 40-100 $\mu \mathrm{m}$ long . . . . R. zambiense

6. Conidia 12-27 septate, 80-165 $\mu$ m long . . . . . R. calongeii

7. Conidia 6-8 septate, $30-50 \times 5-8 \mu \mathrm{m}$, pale brown

R. goidanichii

7. Conidia (4)5-6(7) septate, (25)31-34(45) × (5)6-7(9) $\mu$ m, pale to dark brown.

R. gondwanamycetis

\section{Dematiaceous hyphomycetes first recorded from Spain}

Bactrodesmium abruptum (Berk. \& Broome) E.W. Mason \& S. Hughes in S. Hughes, Can. J. Bot. 36: 738. 1958 (Fig. 3)

Conidiophores sporodochial, usually branched, up to $40 \mu \mathrm{m}$ long. Conidiogenous cells terminal, monoblastic, determinate, cylindrical, 2.5-3.5 $\mu \mathrm{m}$ wide. Conidia clavate, $48-59 \times 11-18 \mu \mathrm{m}, 3-5$ septate, subhyaline to pale brown, with dark bands at the septa, the apical one thicker and black, smooth-walled.

SPAIN. Navarra: Valle del Baztán, Señorío de Bértiz, close to the botanic garden, II-2006, on unidentified dead wood, J. Cano, J. Mena E C. Silvera (FMR 9268). Asturias: Somiedo Natural Park, close to Vigaña, X-2006, on unidentified dead wood, A. Mercado E C. Silvera (FMR 10266). Guipúzcoa: Pagoeta Natural Park, IV2008, on unidentified dead stem, J. Mena \& C. Silvera (FMR 10267).

Bactrodesmium atrum M.B. Ellis, Mycol. Pap. 72: 9. 1959 (Fig. 4)

Conidiophores up to $40 \times 3-4 \mu \mathrm{m}$, unbranched. Conidia clavate, 43-70.5 × 24-38 um, 4-5 septate, dark brown to near black, without thick and dark pigmented bands, smooth-walled.

SPAIN. Navarra: Valle del Baztán, Señorío de Bértiz, close to the botanic garden, II-2006, on unidentified dead wood, J. Cano, J. Mena E C. Silvera (FMR 9215). Guipúzcoa: Pagoeta Natural Park, IV-2008, on unidentified dead wood, J. Mena \& C. Silvera (FMR 10268).

Bactrodesmium spilomeum (Berk. \& Broome) E.W. Mason \& S. Hughes in S. Hughes, Can. J. Bot. 31: 616. 1953 (Fig. 5)

Conidiophore branched, of variable length. Conidia clavate, 30-50 × 8-9 $\mu \mathrm{m}$, mostly 4 septate, pale brown, without thick and dark pigmented bands, smooth-walled.

SPAIN. Navarra: Valle del Baztán, Señorío de Bértiz, close to the botanic garden, II-2006, on unidentified dead wood, J. Cano, J. Mena \& C. Silvera (FMR 9216).

Brachysporium bloxamii (Cooke) Sacc., Syll. fung. (Abellini) 4: 426. 1886 (Fig. 6)

Conidiophores solitary, erect, unbranched, up to $300 \times 4-5 \mu \mathrm{m}$. Conidia ellipsoidal or clavate, $23-30 \times$ 10-11.5 $\mu \mathrm{m}$, mostly 3 septate, basal cell small and paler, other cells larger and brown, smooth-walled.

SPAIN. Asturias: Muniellos Biological Reserve, VI-1999, on unidentified rot wood, M. Calduch \& A. Stchigel (FMR 10269). Navarra: Valle de Roncal, IX-2005, unidentified dead wood, J. Cano E C. Silvera (FMR 9218). Valle del Baztán, Señorío de Bértiz, close to the botanic garden, II-2006, on unidentified dead wood, J. Cano, J. Mena E C. Silvera (FMR 10270). Pontevedra: Monte 
3

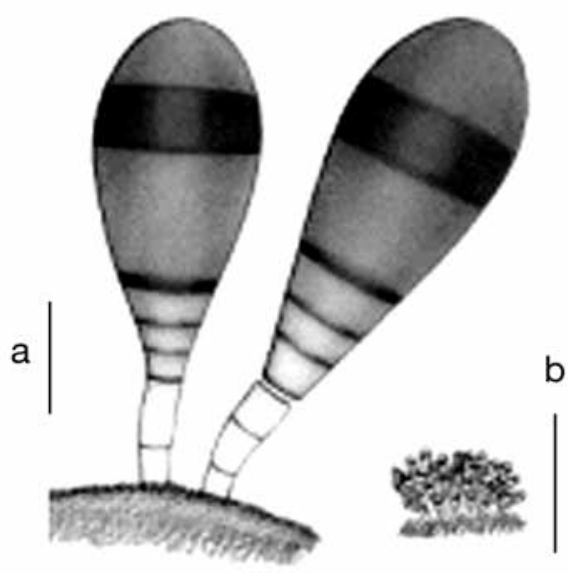

5

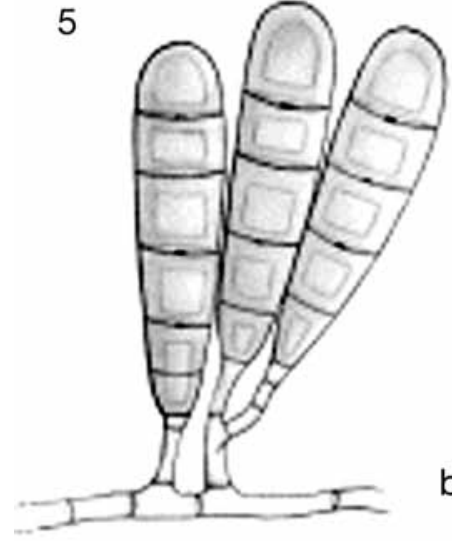

a

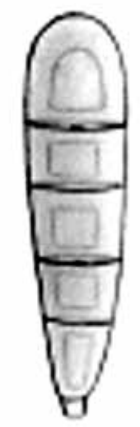

b

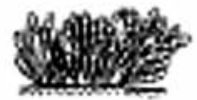

4
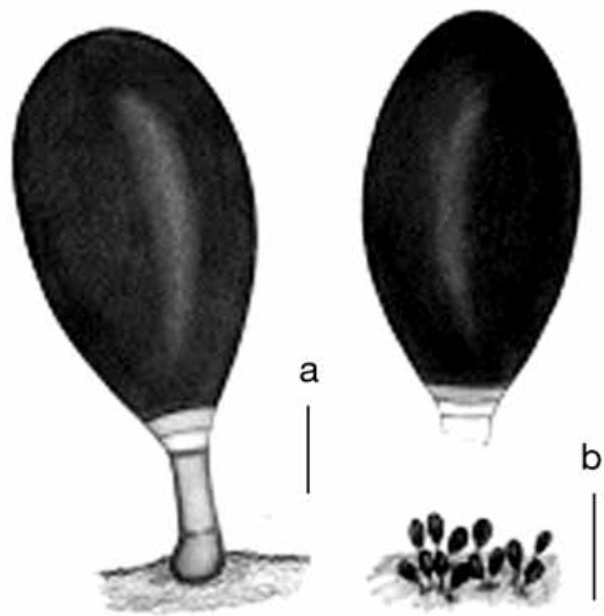

sepere

6
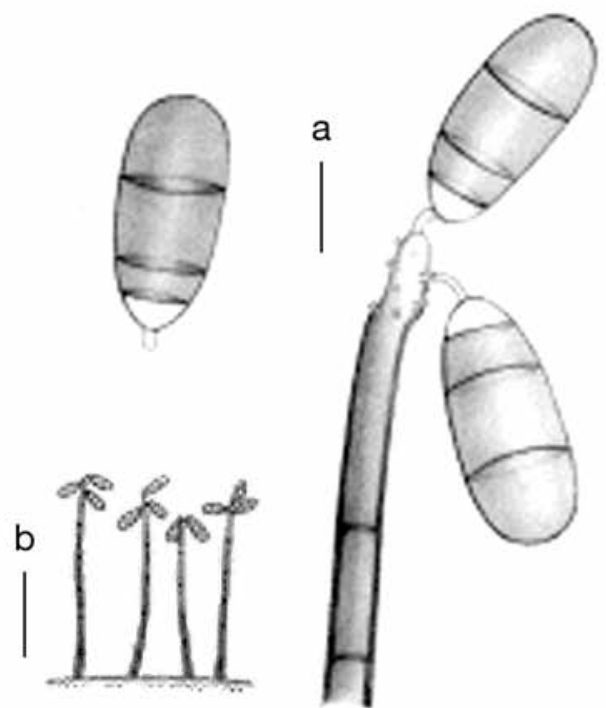

8

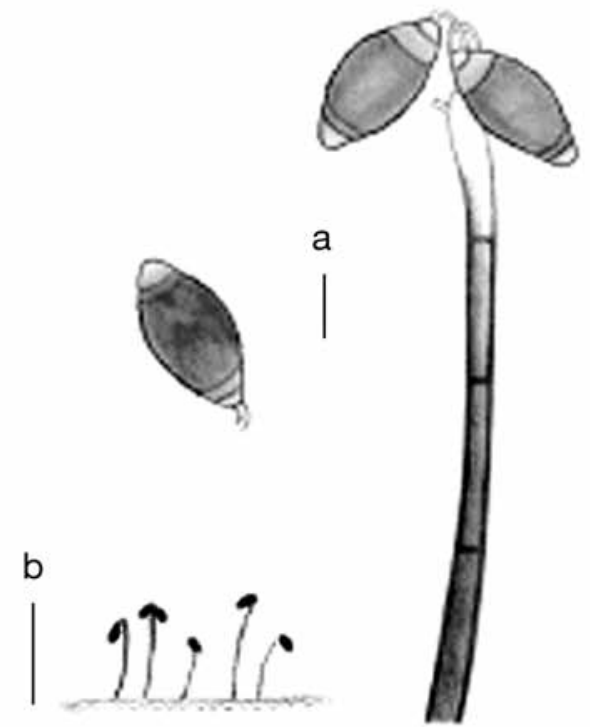

Figs. 3-8. Sketch habit, conidiophores and conidia of: 3, Bactrodesmium abruptum, FMR 9268 (scale bars: $a=10 \mu \mathrm{m} ; b=200 \mu \mathrm{m}$ ). 4, Bactrodesmium atrum, FMR 9215 (scale bars: $a=10 \mu \mathrm{m} ; \mathrm{b}=200$ um). 5, Bactrodesmium spilomeum, FMR 9216 (scale bars: $a=10$ $\mu \mathrm{m} ; \mathrm{b}=100 \mu \mathrm{m}$ ). 6, Brachysporium bloxamii, FMR 9218 (scale bars: $a=10 \mu \mathrm{m} ; \mathrm{b}=100 \mu \mathrm{m}$ ). 7, Brachysporium obovatum, FMR 9220 (scale bars: $a=10 \mu \mathrm{m} ; \mathrm{b}=100 \mu \mathrm{m}) .8$, Brachysporium pendulisporum, FMR 9269 (scale bars: $a=10 \mu \mathrm{m} ; \mathrm{b}=100 \mu \mathrm{m}$ ). 
Aloia, III-2006, on unidentified dead wood, J. Guarro, J. Mena E C. Silvera (FMR 10378).

Brachysporium obovatum Keissl., Syll. fung. (Abellini) 4: 427.1886 (Fig. 7)

Conidiophores up to $130 \times 3.5-5 \mu \mathrm{m}$. Conidia obovate, $19-26.5 \times 11.5-13.5 \mu \mathrm{m}$, mostly 2 septate, usually constricted at septa, basal cell small, subhyaline, other cells larger and pale brown, smooth-walled.

SPAIN. Navarra: Valle del Baztán, Señorío de Bértiz, close to the botanic garden, II-2006, on unidentified dead wood, J. Cano, J. Mena E C. Silvera (FMR 9220). Guipúzcoa: Pagoeta Natural Park, IV2008, on unidentified dead wood, J. Mena \& C. Silvera (FMR 10271).

Brachysporium pendulisporum S. Hughes, Can. J. Bot. 33: 266. 1955 (Fig. 8)

Conidiophores up to $250 \times 9-14 \mu \mathrm{m}$. Conidia limoniform, 28-39 × 12-17 $\mu \mathrm{m}$, mostly 4 septate, central cell very large and dark brown, basal and apical cells smaller and paler, often verruculose.

SPAIN. Navarra: Valle del Baztán, Señorío de Bértiz, close to the botanic garden, II-2006, on unidentified dead wood, J. Cano, J. Mena \& C. Silvera (FMR 9269).

Camposporium cambrense S. Hughes, Mycol. Pap. 36: 11.1951 (Fig. 9)

Conidiophores solitary, unbranched, up to $60 \mu \mathrm{m}$ long. Conidiogenous cells terminal, polyblastic, sympodial, denticulate, cylindrical, 5-6 $\mu \mathrm{m}$ wide. Conidia up to 12 septate, cylindrical, 70-95 $\times 9-10 \mu \mathrm{m}$, rounded at the end, and with a basal hilum up to $1.5 \mu \mathrm{m}$ wide, pale brown, smooth-walled, the apical cell may bear a hyaline, septate appendage not seen in the present collection.

SPAIN. Navarra: Valle del Baztán, Señorío de Bértiz, close to the botanic garden, II-2006, on unidentified dead wood, J. Cano, J. Mena EC. Silvera (FMR 10272).

Ceratosporium fuscescens Schwein., Trans. Am. phil. Soc., Ser. 2, 4: 300. 1832 (Fig. 10)

Conidiophores inconspicuous. Conidiogenous cells intercalary, monoblastic, denticulate, denticles up to $2 \mu \mathrm{m}$ wide. Conidia composed of three elongate arms, arising from a $15-19 \times 15 \mu \mathrm{m}$, pyriform basal cell; each arm 6-10 septate, $88-135 \times 15-23 \mu \mathrm{m}$, pale brown, smooth-walled.

SPAIN. Navarra: Valle del Baztán, Señorío de Bértiz, close to the botanic garden, II-2006, on unidentified dead wood, J. Cano, J. Mena \& C. Silvera (FMR 9222).

Cryptocoryneum condensatum (Wallr.) E.W. Mason \& S. Hughes in Walsh \& Rimington, Nat. Hist. Scarborough Distr. 1: 161. 1953 (Fig. 11)
Conidiophores sporodochial, unbranched, subhyaline or pale brown, up to $60 \mu \mathrm{m}$ long. Conidiogenous cells terminal, monoblastic, determinate, cylindrical, 3-5 $\mu \mathrm{m}$ wide. Conidia solitary, 30-50 × 20-30 $\mu \mathrm{m}$, branched, consisting of a several, small, apical dark cells, from which are borne 5-8 pendulous arms; arms mostly 6 septate, $30-50 \times 5-6 \mu \mathrm{m}$, subhyaline to pale brown, smooth-walled.

SPAIN. Navarra: Valle del Baztán, Señorío de Bértiz, close to the botanic garden, II-2006, on unidentified dead wood, J. Cano, J. Mena E C. Silvera (FMR 9229).

Cryptocoryneum rilstonii M.B. Ellis, Mycol. Pap. 131: 2. 1972 (Fig. 12)

Conidiophores pale brown, very slender, up to $40 \times$ 1-3 $\mu \mathrm{m}$. Conidia solitary, $28-39 \times 22-34 \mu \mathrm{m}$, with apical
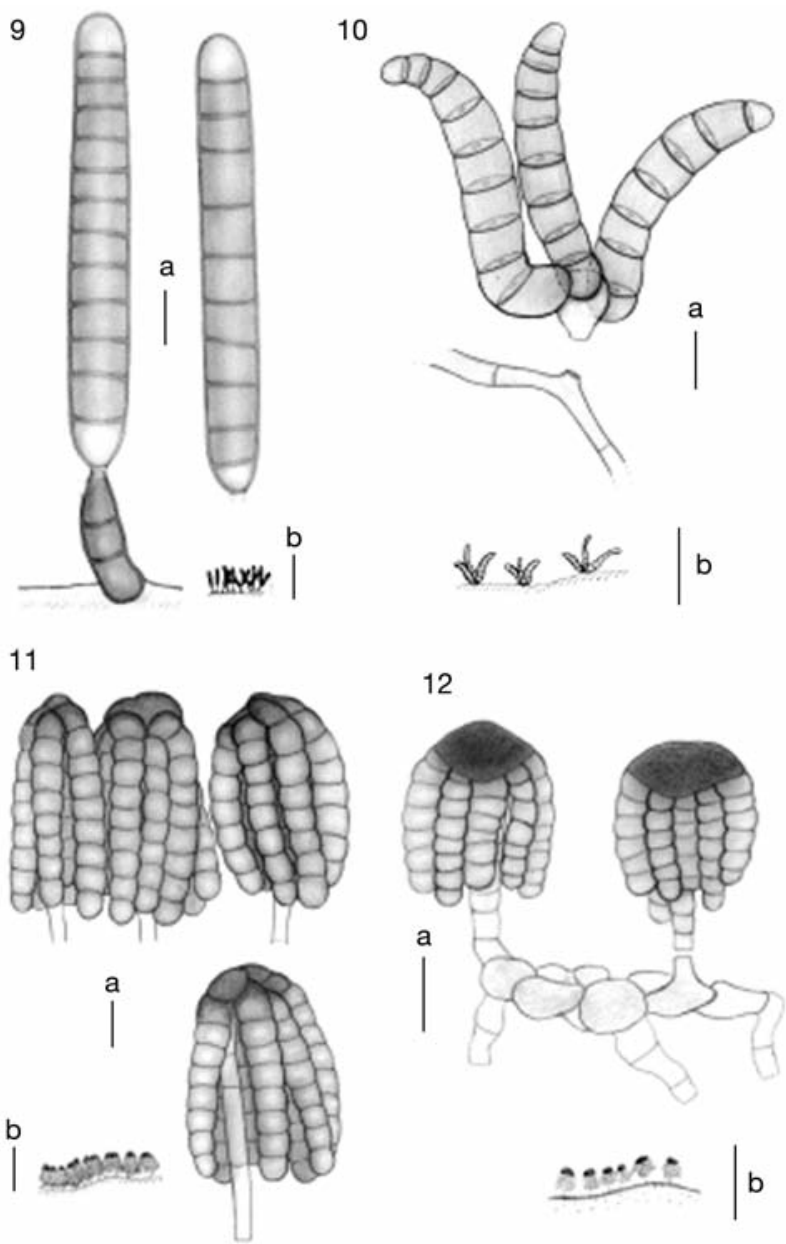

Figs. 9-12. Sketch habit, conidiophores and conidia of: 9, Camposporium cambrense, FMR 10272 (scale bars: $a=10 \mu \mathrm{m} ; \mathrm{b}=$ $200 \mu \mathrm{m})$. 10, Ceratosporium fuscescens, FMR 9222 (scale bars: $\mathrm{a}=20 \mu \mathrm{m} ; \mathrm{b}=200 \mu \mathrm{m})$. 11, Cryptocoryneum condensatum, FMR 9229 (scale bars: $a=10 \mu \mathrm{m} ; \mathrm{b}=100 \mu \mathrm{m}) .12$, Cryprocoryneum rilstonii, FMR 9230 (scale bars: $\mathrm{a}=10 \mu \mathrm{m} ; \mathrm{b}=100 \mu \mathrm{m}$ ). 
black cells and 6-9 pendulous arms; arms 4-6 septate, $21-30 \times 5-6 \mu \mathrm{m}$, subhyaline to pale brown, smoothwalled.

SPAIN. Navarra: Valle del Baztán, Señorío de Bértiz, close to the botanic garden, II-2006, on unidentified dead wood, J. Cano, J. Mena \& C. Silvera (FMR 9230). Pontevedra: Monte Aloia, III2006, on unidentified dead leaf, J. Guarro, J. Mena \& C. Silvera (FMR 10273).

Ellisembia adscendens (Berk.) Subram., Proc. Indian natn. Sci. Acad., Part B Biol. Sci. 58: 183. 1992 (Fig. 13)

Conidiophores solitary, erect or flexuous, septate, unbranched, brown, smooth-walled, up to $30 \times 7-8$ $\mu \mathrm{m}$. Conidiogenous cells terminal, monoblastic, without percurrent proliferations. Conidia 11-24 pseu-

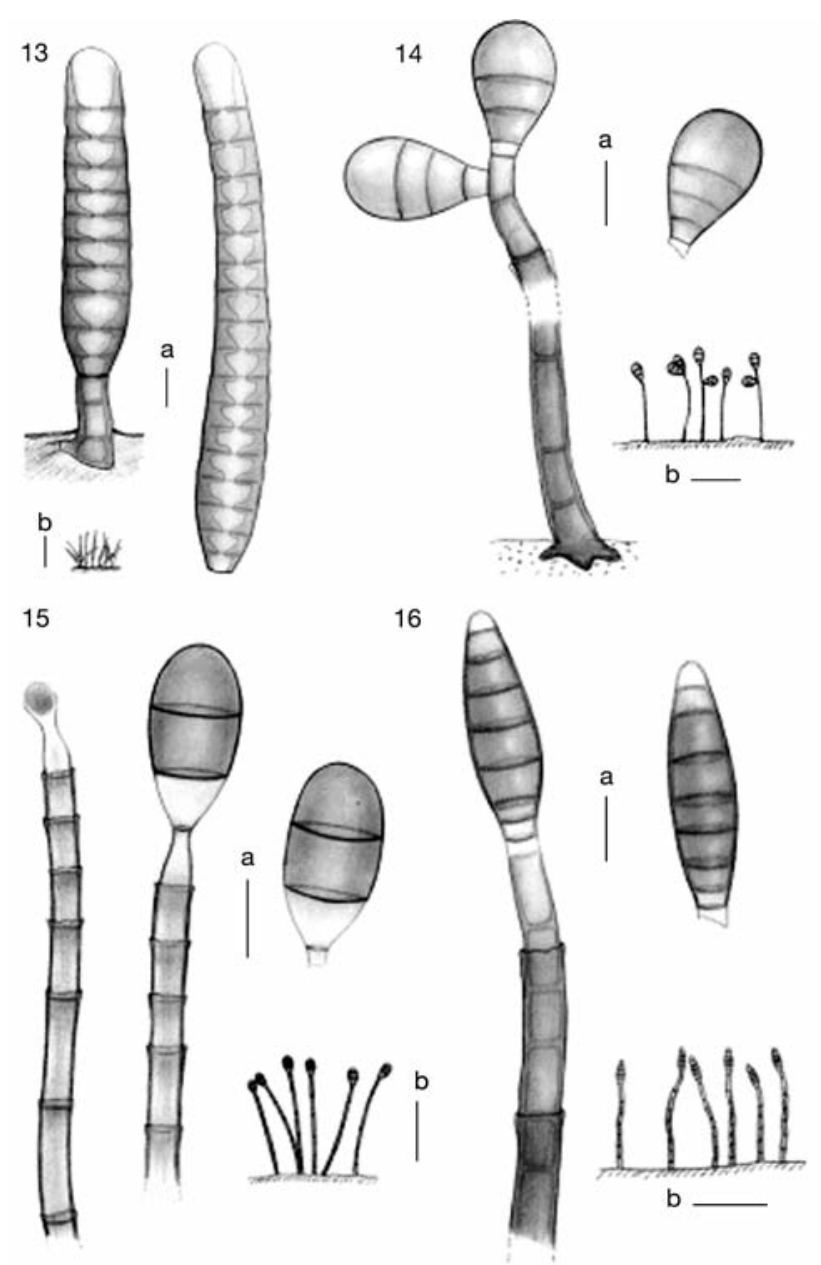

Figs. 13-16. Sketch habit, conidiophores and conidia of: 13, Ellisembia adescendens, FMR 9736 (scale bars: $a=10 \mu$ m; $b=200$ um). 14, Endophragmiella boothii, FMR 9239 (scale bars: $a=10$ $\mu \mathrm{m} ; \mathrm{b}=100 \mu \mathrm{m}$ ). 15, Endophragmiella oblonga, FMR 9241 (scale bars: $a=10 \mu \mathrm{m} ; \mathrm{b}=100 \mu \mathrm{m})$. 16, Endophragmiella socia, FMR 9242 (scale bars: $a=10 \mu \mathrm{m} ; b=100 \mu \mathrm{m}$ ). doseptate, slightly or distinctly constricted at the septa, cylindrical, obclavate, rostrate, up to $200 \mu \mathrm{m}$ long, $12-15 \mu \mathrm{m}$ wide at the broadest part, base of $6-8 \mu \mathrm{m}$ wide, brown but paler at the apex, smooth-walled.

SPAIN. Navarra: Valle del Baztán, Señorío de Bértiz, close to the botanic garden, II-2006, on unidentified dead wood, J. Cano, J. Mena \& C. Silvera (FMR 9736).

Endophragmiella boothii (M.B. Ellis) S. Hughes, N. Z. Jl Bot. 17: 147. 1979 (Fig. 14)

Conidiophores usually solitary, unbranched, septate, brown, up to $120 \times$ 3.5-4.5 $\mu \mathrm{m}$. Conidiogenous cells terminal, monoblastic, percurrent, cylindrical. Conidia predominantly 3 septate, oblonga-ellipsoidea, 19-23 $\times$ $11-13 \mu \mathrm{m}$, with a basal frill up to $1.5 \times 3.5-4 \mu \mathrm{m}$, pale brown to golden brown, smooth-walled.

SPAIN. Navarra: Valle del Baztán, Señorío de Bértiz, close to the botanic garden, II-2006, on unidentified dead wood, J. Cano, J. Mena \& C. Silvera (FMR 9239).

Endophragmiella oblonga (Matsush.) S. Hughes, N. Z. J1 Bot. 17: 152. 1979 (Fig. 15)

Conidiophores up to $220 \times 7-8 \mu \mathrm{m}$. Conidia predominantly 2 septate, oblonga-ellipsoidea, 23-25 $\times$ 11-13 $\mu \mathrm{m}$, with a basal frill up to $1.5 \times 2-2.5 \mu \mathrm{m}$, pale brown to brown, basal cell paler, smooth-walled.

SPAIN. Navarra: Valle del Baztán, Señorío de Bértiz, close to the botanic garden, II-2006, on unidentified dead wood, J. Cano, J. Mena E C. Silvera (FMR 9241).

Endophragmiella socia (M.B. Ellis) S. Hughes, N. Z. J1 Bot. 17: 153. 1979 (Fig. 16)

Conidiophores up to $220 \times 6.5-7.5 \mu \mathrm{m}$. Conidia predominantly 7-8 septate, navicular, 30-40 × 11-15 $\mu \mathrm{m}$, with a scar 5-6 $\mu \mathrm{m}$ wide, pale brown to brown, terminal cells paler, smooth-walled.

SPAIN. Navarra: Valle del Baztán, Señorío de Bértiz, close to the botanic garden, II-2006, on unidentified dead wood, J. Cano, J. Mena \& C. Silvera (FMR 9242).

Endophragmiella valdiviana (Speg.) S. Hughes, N.Z. J1 Bot. 17: 157.1979 (Fig. 17)

Conidiophores up to $230 \times 7-9 \mu \mathrm{m}$. Conidia mostly 7 septate, navicular, 39-49 $\times 11-13 \mu \mathrm{m}$, with a scar 7-8 $\mu \mathrm{m}$ wide, brown, paler in the both end cells, smooth-walled.

SPAIN. Navarra: Valle del Baztán, Señorío de Bértiz, close to the botanic garden, II- 2006, on unidentified dead wood, J. Cano, J. Mena \& C. Silvera (FMR 9744).

Repetophragma goidanichii (Rambelli) W.P. Wu in Wu \& Zhuang, 2005 (Fig. 18) 

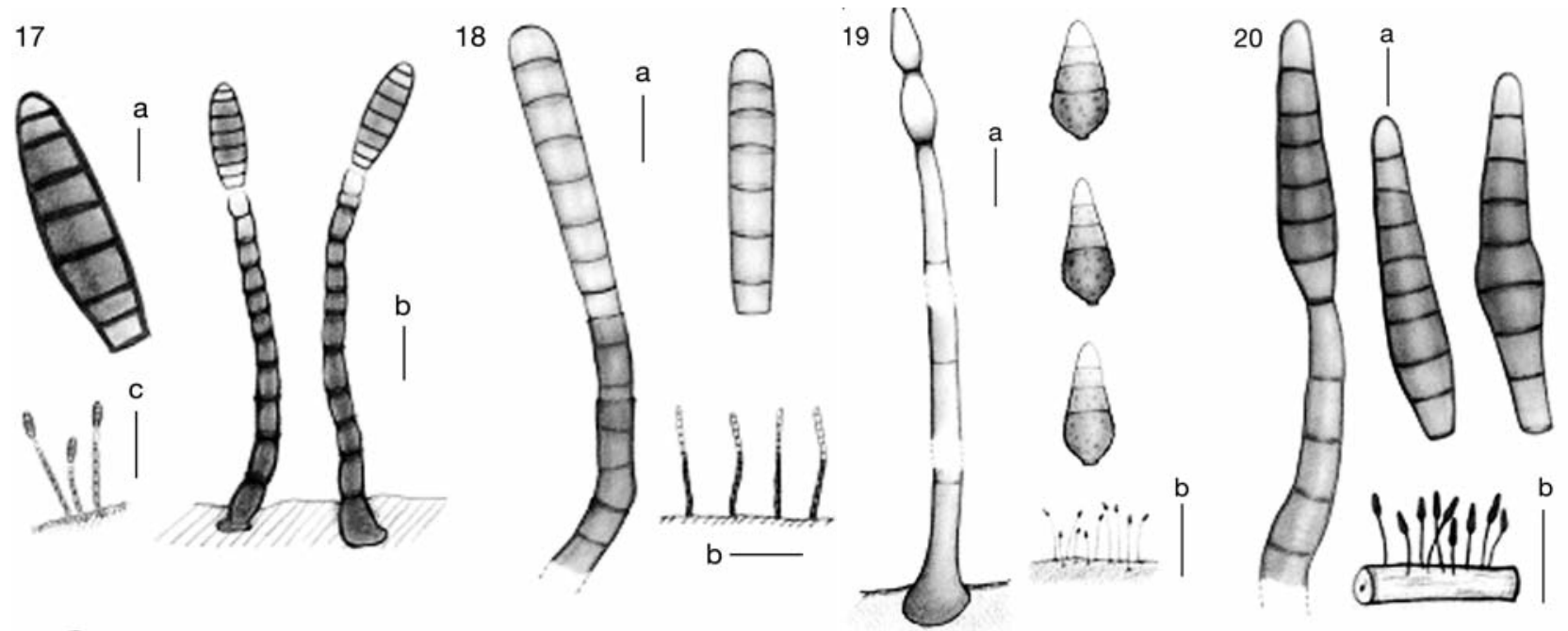

Figs. 17-20. Sketch habit, conidiophores and conidia of: 17, Endophragmiella valdiviana, FMR 9744 (scale bars: $a=10 \mu \mathrm{m} ; \mathrm{b}=25 \mu \mathrm{m}$; $c=100 \mu \mathrm{m}$ ). 18, Repetophragma goidanichii, FMR 9260 (scale bars: $a=10 \mu \mathrm{m} ; \mathrm{b}=100 \mu \mathrm{m}$ ). 19, Sporidesmium cookei, FMR 10274 (scale bars: $a=10 \mu \mathrm{m} ; \mathrm{b}=200 \mu \mathrm{m}) .20$, Sporidesmium fragilissimum, FMR 10275 (scale bars: $a=10 \mu \mathrm{m} ; \mathrm{b}=200 \mu \mathrm{m}$ ).

Conidiophores solitary, erect or flexuous, septate, brown to dark brown, up to $140 \times 4.5-6 \mu \mathrm{m}$. Conidiogenous cells with several annellating percurrent proliferations. Conidia 6-8 euseptate, cylindrical to subcylindrical, 38-50 × 5.5-7 $\mu \mathrm{m}$, pale brown, smooth-walled.

SPAIN. Pontevedra: Monte Aloia, III-2006, on unidentified dead leaf, J. Guarro, J. Mena \& C. Silvera (FMR 9260). Zamora: Moveros, IX-2007, on Quercus suber dead leaf, J. Capilla, R. Castañeda \& C. Silvera (FMR 9745).

Sporidesmium cookei (S. Hughes) M.B. Ellis, Mycol. Pap. 70: 48. 1958 (Fig. 19)

Conidiophores solitary, erect, unbranched, up to $118 \times 3.5-4 \mu \mathrm{m}$, brown. Conidiogenous cells with 1-2 lageniform or ampulliform pecurrent proliferations. Conidia 3 septate, obpyriform or obclavate, 17-23 $\times$ 7-10 $\mu \mathrm{m}$ wide at the broadest part, the basal cells are roughened, brown, apical cells paler and smoother, rounded at the apex, truncate at the base.

SPAIN. Vizcaya: Urkiola Natural Park, V-2008, on unidentified dead wood, J. Mena and C. Silvera (FMR 10274).

Sporidesmium fragilissimum (Berk. \& M.A. Curtis) M.B. Ellis, Mycol. Pap. 70: 55. 1958 (Fig. 20)

Conidiophores solitary, erect, unbranched, cylindrical, up to $85 \times 7-9 \mu \mathrm{m}$ wide at the broadest part. Conidiogenous cells usually without proliferations. Conidia 5-7 septate, obclavate, 37-56 × 9-11 $\mu$ m wide at the broadest part, with apex rounded, brown to pale brown, apical cells paler and smooth, verruculose towards the base.

SPAIN. Vizcaya: Urkiola Natural Park, V-2008, on unidentified dead wood, J. Mena \& C. Silvera (FMR 10275).

\section{Acknowledgements}

This study was supported by the grants from the Ministry of Science and Technology of Spain CGL 2008-04226 and CGL 2009-08698.

\section{References}

Iturriaga, T., Hawksworth, D.L., \& Crane, J.L. 2008. 'Sporidesmium' lichenicola sp. nov., a new lichenicolous fungus on Leptogium from Venezuela. Mycologia 100: 392-396.

Shenoy, D.B., Jeewon, R., Wu, W.P., Bhat, D.J., \& Hyde, K.D. 2006. Ribosomal and RPB2 DNA sequence analyses suggest that Sporidesmium and morphologically similar genera are polyphyletic. Mycological Research 110: 916-928.

Subramanian, C.V. 1992. A reassessment of Sporidesmium (hyphomycetes) and some related taxa. Proceedings of the Indian National Science Academy B 58: 179-190.

Wu, W.P., \& Zhuang, W. 2005. Sporidesmium, Endophragmiella and related genera from China. Institute of Microbiology The Chinese Academy of Sciences Zhongguan, Beijing. Fungal Diversity Press Production Editor K.D. Hyde Fungal Diversity Research Series 15. 